\title{
Inter-satellite Ranging Augmented GPS Relative Navigation for Satellite Formation Flying
}

\author{
Yang Yang ${ }^{1,2}$, Yong $\mathrm{Li}^{2}$, Chris Rizos ${ }^{2}$, Andrew G. Dempster ${ }^{3}$ \\ and Xiaokui Yue ${ }^{1}$ \\ ${ }^{1}$ (School of Astronautics, Northwestern Polytechnical University, China) \\ ${ }^{2}$ (School of Civil \& Environmental Engineering, \\ University of New South Wales, Australia) \\ ${ }^{3}$ (The Australian Centre for Space Engineering Research, UNSW, Australia) \\ (E-mail: yiyinfeixiong@gmail.com)
}

\begin{abstract}
An Augmented Relative Navigation System (ARNS) is proposed for autonomous satellite formation flying in low-Earth-orbit (LEO). Inter-satellite ranging systems such as those based on radio frequency transmissions can provide additional observation information, e.g. inter-satellite distance measurement, which can be used to increase the Global Positioning System (GPS) stand-alone observation dimension, or treated as a non-linear equality constraint within a smoothly-constrained Kalman filter. Both approaches are implemented in the proposed ARNS described in this paper. An innovative phase integer ambiguity fixing and feedback scheme is implemented to increase the ambiguity fix rate of the GPS carrier phase measurements. A set of Gravity Recovery and Climate Experiment (GRACE) flight data is used to test and validate the relative navigation performance of the proposed methods. Results indicate that the augmented system can improve relative positioning accuracy by an order of magnitude.
\end{abstract}

\section{KEYWORDS}

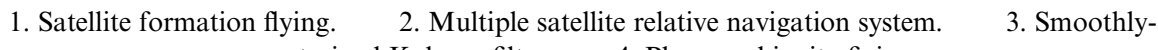
constrained Kalman filter. 4. Phase ambiguity fixing.

Submitted: 8 October 2013. Accepted: 3 November 2013. First published online: 26 November 2013.

1. INTRODUCTION. Satellite formation flying is one of the typical forms of a so-called Distributed Space System, in which more than two spacecraft are flying in a coordinated manner to fulfil a particular space mission. The formation flying of spacecraft to replace a single large satellite will provide opportunities for a number of future applications, including synthetic apertures for high-resolution interferometry missions. Such missions require precise relative satellite state (position and velocity) information. Hence relative navigation is one of the primary tasks in a satellite formation flying mission. 
Table 1. Relative positioning performance using real data for several LEO formation flying missions.

\begin{tabular}{lllll}
\hline Mission & \multicolumn{1}{c}{ Measurements } & $\begin{array}{c}\text { Nominal } \\
\text { separation }\end{array}$ & $\begin{array}{c}\text { Process } \\
\text { strategy }\end{array}$ & \multicolumn{1}{c}{ Positioning precision } \\
\hline GRACE & Dual-frequency GPS & $220 \mathrm{~km}$ & Post processing & $1.56 \mathrm{~mm}$ 3D std dev (Kroes 2006) \\
GRACE & Dual-frequency GPS & $260 \mathrm{~km}$ & Near-real-time & $4.2 \mathrm{~cm} \mathrm{3D} \mathrm{rms} \mathrm{(Tancredi} \mathrm{et} \mathrm{al.} \mathrm{2013)}$ \\
TanDEM-X & Dual-frequency GPS & $20 \mathrm{~km}$ & Post processing & $\sim 1 \mathrm{~mm}$ 3D std dev (Jäggi et al., 2012) \\
PRISMA & Single-frequency GPS & $0 \sim 30 \mathrm{~km}$ & Real-time & $5 \mathrm{~cm}$ 3D rms (D'Amico et al., 2013)
\end{tabular}

Traditionally external observations from ground stations have been used for satellite orbit determination. However, more and more space missions benefit from the use of an autonomous navigation system, which can estimate a satellite's state using the measurements available on board. Since the beginning of the 1990s, the U.S. Global Positioning System (GPS) has been used for spacecraft navigation, providing high precision orbit information with minimum ground intervention.

The differential GPS technique can produce sub-centimetre-level relative positioning precision and accuracy in software or/and hardware-in-the-loop simulations, and centimetre-level precision in (near) real time for low Earth orbit (LEO) satellite formation flying missions. One significant issue affecting relative navigation performance is the inter-satellite distance. For formations with large separation, e.g. over $100 \mathrm{~km}$, even though most common GPS errors can be eliminated by using the doubledifferenced observables, ionospheric delay residuals may still be significant. Moreover, the common-in-view GPS satellites change rapidly, which will degrade the success rates of integer ambiguity resolution and lead to solution gaps when there are insufficient double-differenced observables (Mohiuddin and Psiaki, 2005; Tancredi et al., 2010; Montenbruck and D'Amico, 2013). As a consequence, the navigation performance will deteriorate significantly. Relative positioning performance for some formation flying missions are summarised in Table 1.

Given the wide range of formation flying missions, a single navigation system will not necessarily be able to meet all functional and operational requirements. For example, as introduced in the previous section, some weakness still exists in GPS standalone baseline determination for satellite formation flying applications. However, inter-satellite ranging observations are sometimes available for satellite formation flying missions to provide robust and autonomous measurements (Tapley et al., 2004; Montenbruck et al., 2008). Inter-satellite ranging systems are categorised as optical, radio, laser, or laser interferometry (Renga et al., 2013). Radio signal measurement is the most mature class of technology.

The Gravity Recovery and Climate Experiment (GRACE) mission has successfully demonstrated the use of inter-satellite ranging between two satellites to aid determination of the Earth's gravitational field in a precise manner (Tapley et al., 2004). The relative distance between the satellites is measured with a resolution of $10 \mu \mathrm{m}$ using a $\mathrm{K} / \mathrm{Ka}$-band ranging (KBR) system, which is a dual one-way ranging instrument. A GRACE follow-on mission is planned to launch in 2017 (Sheard et al., 2012). It is planned to use microwave ranging as the primary instrument for inter-satellite distance measurements, however laser interferometry has been proposed as an alternative system, able to achieve improved inter-satellite ranging precision. Another mission known as PRISMA also benefits from use of a radio frequency (RF) 
Table 2. Inter-satellite ranging systems for some LEO formation flying missions.

\begin{tabular}{llc}
\hline Name & \multicolumn{1}{c}{ Type } & Positioning Accuracy \\
\hline GRACE & Microwave ranging & $10 \mu \mathrm{m}$ \\
PRISMA & Microwave ranging \& vision-based sensor & $0 \cdot 1 \mathrm{~m}$ \\
GRACE-II & Microwave ranging \& laser interferometry & $50 \mathrm{~nm}$ \\
\hline
\end{tabular}

metrology subsystem for relative positioning (Montenbruck et al., 2008). A summary of these systems is given in Table 2 .

In these flight missions the inter-satellite ranging instruments have been applied to measure the inter-satellite baseline directly, or with the aid of GPS. An augmented relative navigation system (ARNS) is one that combines GPS measurements with inter-satellite range measurements, which is able to provide a sufficient number of measurements, as well as more rapid and stable navigation filter solutions. In this navigation system, the inter-satellite range measurement is treated as a new observation, which can be combined with the original GPS stand-alone measurements. Alternatively, the baseline measurement can be used as a non-linear equality constraint. A number of approaches to applying equality constraints with a Kalman filter framework (Simon, 2010) can also be applied to the ARNS. In particular, a smoothlyconstrained Kalman filter (SCKF) (De Geeter et al., 1997) can be used.

An augmented relative navigation system for satellite formation flying is described in this paper. Section 2 presents the functional models and the extended Kalman filter architecture for the stand-alone GPS-based kinematic relative navigation system. Section 3 introduces two approaches to incorporating the extra measurements from the inter-satellite ranging instrument into the ARNS. A phase ambiguity fixing and feedback scheme is investigated in Section 4. A set of fight data from the GRACE mission was used to test and validate the ARNS performance.

2. GPS-BASED KinEMATIC RELATIVE NAVIGATION. The double-differenced GPS observables are widely used to obtain high accuracy baseline solutions. By taking differences between GPS satellites and two receivers the doubledifferenced observation equation can be written as follows:

$$
\begin{aligned}
& \delta P R_{r_{c d}}^{s_{i, j}}=-\operatorname{los}_{r_{d}, 0}^{s_{i j}} \delta \boldsymbol{r}_{r_{c d}}-\dot{\rho}_{r_{c}}^{s_{i}} \delta c l_{r_{c}}+\dot{\rho}_{r_{c}}^{s_{j}} \delta c l_{r_{c}}+\dot{\rho}_{r_{d}}^{s_{i}} \delta c l_{r_{d}}-\dot{\rho}_{r_{d}}^{s_{j}} \delta c l_{r_{d}}+I_{r_{c d}}^{s_{i j, f}}+v_{r_{d}}^{s_{i j, f}} \\
& \delta C P_{r_{c d}}^{s_{i, j}}=-\operatorname{los}_{r_{d}, 0}^{s i j} \delta \boldsymbol{r}_{r_{c d}}-\dot{\rho}_{r_{c}}^{s_{i}} \delta c l_{r_{c}}+\dot{\rho}_{r_{c}}^{s_{j}} \delta c l_{r_{c}}+\dot{\rho}_{r_{d}}^{s_{i}} \delta c l_{r_{d}}-\dot{\rho}_{r_{d}}^{s_{j}} \delta c l_{r_{d}}-I_{r_{c d}}^{s_{i j, f}}+\lambda^{f} N_{r_{c d}}^{s_{i j, f}}+v_{r_{c d}}^{s_{i j}}
\end{aligned}
$$

where the superscript $s$ and $f$ indicate the GPS satellite and the signal frequency respectively, and subscript $r$ indicates the receiver. The terms in the equations are:

$\delta \quad$ Increment operator: $\delta()=0-0_{0}$

$P R, C P \quad$ Code phase and carrier phase observations [m]

$\rho_{P R}, \rho_{C P} \quad$ Geometric distance between receiver and GPS satellites [m]

I Ionospheric delay [m]

cl Clock error [s]

$\lambda^{f} \quad$ Signal wavelength at frequency $f[\mathrm{~m}]$

$N \quad$ Carrier phase integer ambiguity [cycle]

$v \quad$ Unmodelled code phase and carrier phase errors [m] 
As indicated in the above models, the terms associated with the receiver clock errors remain and need to be accounted for. A typical GPS receiver with a pulse per second output can provide an accuracy of between 100 nanoseconds and 1 microsecond. In the high dynamic motion of a spacecraft where the multiplier $\dot{\rho}_{r}^{s}$ is large, e.g. about $7.5 \mathrm{~km} / \mathrm{s}$ on a $500 \mathrm{~km}$ circular orbit, the magnitude of these terms will be at the millimetre level and can be ignored. Common expressions of the double-differenced models are the same as those used in terrestrial scenarios:

$$
\begin{aligned}
& \delta P R_{r_{c d}}^{s_{i j}, f}=-\operatorname{los}_{r_{d}, 0}^{s_{i j}} \delta \boldsymbol{r}_{r_{c d}}+I_{r_{c d}}^{s_{i j}, f}+v_{r_{c d}}^{s_{i j}, f} \\
& \delta C P_{r_{c d}}^{s_{i j}, f}=-\operatorname{los}_{r_{d}, 0}^{s i j} \delta \boldsymbol{r}_{r_{c d}}-I_{r_{c d}}^{s_{i j}, f}+\lambda^{f} N_{r_{c d}}^{s_{i j}, f}+v_{r_{c d}}^{s_{i j}}
\end{aligned}
$$

The extended Kalman filter (EKF) can be used to resolve the optimal estimation problem of non-linear systems, which are linearized around the current estimate and its covariance. The relative navigation system employs a common EKF for estimating the relative states of two spacecraft. The single point positioning (SPP) approach is used to estimate the absolute orbits and receiver clock errors, which are then treated as the initial approximate values for linearization of the system of equations. A several metre-level of accuracy for absolute orbit determination is sufficient. The unknown parameters to be estimated are:

$$
x=\left(r_{x c}, r_{y c}, r_{z c}, c l_{c}, r_{x d}, r_{y d}, r_{z d}, c l_{d}, \mathbf{B}, v T E C_{c}, v T \dot{E} C_{c}, v T E C_{d}, v T \dot{E} C_{d}, \mathbf{N}\right)^{T}
$$

The subscript $c$ and $d$ designate the "chief" and "deputy" spacecraft, respectively, $\left[r_{x}, r_{y}, r_{z}\right]$ are the absolute position components in the Earth-Centred-Earth-Fixed (ECEF) coordinate system, $c l$ denotes the receiver clock error, $\mathbf{B}=\left[B_{x}, B_{y}, B_{z}\right]$ is the baseline vector, $v T E C$ and $v T \dot{E} C$ are the vertical total electron content and its rate, respectively. $\mathbf{N}=\left(N_{c d}^{1, L_{1}}, N_{c d}^{2, L_{1}}, \cdots N_{c d}^{i, L_{1}}, N_{c d}^{1, L_{2}}, N_{c d}^{2, L_{2}}, \cdots N_{c d}^{i, L_{2}}\right)^{T}$ denotes the singledifferenced carrier phase ambiguities. Only $\left(\mathbf{B}, v T E C_{c}, v T \dot{E} C_{c}, v T E C_{d}, v T \dot{E} C_{d}, \mathbf{N}\right)^{T}$ are estimated as states in the EKF. The formulation of the first order ionospheric error with respect to $v T E C$ can be expressed as:

$$
I_{r}^{s}=\frac{40 \cdot 3}{f^{2}} \cdot m \cdot v T E C
$$

where $m$ is the mapping function and $f$ denotes the carrier frequency. Since GPS observations by space borne receivers are all measured within the ionosphere rather than underneath it, the ionospheric pierce point model used for terrestrial receivers cannot be applied directly. The Lear mapping function (Leung and Montenbruck, 2005; van Barneveld et al., 2008) is commonly used, which is dependent on the elevation angle:

$$
m(E)=\frac{2 \cdot 037}{\sqrt{\sin ^{2} E+0.076}+\sin E}
$$

There are two steps in the EKF process: state prediction and state propagation. When only the kinematic relative navigation approach is applied, the baseline components can be propagated using the identify matrix without any a priori knowledge of relative motion. $v T E C$ is modelled to change with a constant rate within a short time interval. Based on this assumption, its dynamics can be modelled as a second-order random walk process. In the state prediction step the relative states 
propagate as follows:

$$
\begin{aligned}
& \hat{\mathbf{B}}_{k+1}^{-}=\hat{\mathbf{B}}_{k}^{+} \\
& v T \hat{E} C_{c, k+1}^{-}=v T \hat{E} C_{c, k}^{+}+v T \hat{\dot{E}} C_{c, k}^{+} \cdot\left(t_{k+1}-t_{k}\right) \\
& v T \hat{E} C_{d, k+1}^{-}=v T \hat{E} C_{d, k}^{+}+v T \hat{\dot{E}} C_{d, k}^{+} \cdot\left(t_{k+1}-t_{k}\right) \\
& \left(v T \hat{\dot{E}} C_{c}, v T \hat{\dot{E}} C_{d}, \mathbf{N}\right)_{k+1}^{-}=\left(v T \hat{\dot{E}} C_{c}, v T \hat{\dot{E}} C_{d}, \mathbf{N}\right)_{k}^{+}
\end{aligned}
$$

After the ionosphere error is modelled with respect to vTEC by means of the Lear mapping function, the double-differenced observables can be finally expressed as:

$$
\begin{aligned}
& \delta P R_{r_{c d}}^{s_{i j}, L_{1}}=-\operatorname{los}_{r_{d}, 0}^{s_{i j}} \mathbf{B}+\frac{1}{f_{L_{1}}^{2}}\left(m_{d}^{i j} v T E C_{d}-m_{c}^{i j} v T E C_{c}\right)+v_{P R_{c d}}^{s_{i j}, L_{1}} \\
& \delta P R_{r_{c d}}^{s_{i j}, L_{2}}=-\operatorname{los}_{r_{d}, 0}^{s_{i j}} \mathbf{B}+\frac{1}{f_{L_{2}}^{2}}\left(m_{d}^{i j} v T E C_{d}-m_{c}^{i j} v T E C_{c}\right)+v_{P R_{c d}}^{s_{i j}, L_{2}} \\
& \delta C P_{r_{c d}}^{s_{i j}, L_{1}}=-\operatorname{los}_{r_{d}, 0}^{s i j} \mathbf{B}-\frac{1}{f_{L_{1}}^{2}}\left(m_{d}^{i j} v T E C_{d}-m_{c}^{i j} v T E C_{c}\right)+\lambda^{f}\left(N_{r_{c d}}^{s_{i}, L_{1}}-N_{r_{c d}}^{s_{j}, L_{1}}\right)+v_{C P_{c d}}^{s_{i j}, L_{1}} \\
& \delta C P_{r_{c d}}^{s_{i j}, L_{2}}=-\operatorname{los}_{r_{d}, 0}^{s i j} \mathbf{B}-\frac{1}{f_{L_{2}}^{2}}\left(m_{d}^{i j} v T E C_{d}-m_{c}^{i j} v T E C_{c}\right)+\lambda^{f}\left(N_{r_{c d}}^{s_{i}, L_{2}}-N_{r_{c d}}^{s_{j}, L_{2}}\right)+v_{C P_{c d}}^{s_{i j}, L_{2}}
\end{aligned}
$$

3. AUGMENTED RELATIVE NAVigation System. Two approaches can be used to implement the augmented navigation system with baseline measurements: system re-parameterisation and a smoothly-constrained Kalman filter (SCKF).

3.1. System re-parameterisation. RF inter-satellite observations may be range and carrier phase:

$$
\begin{aligned}
& R_{B L}=\rho_{B L}+E_{r}+\sigma_{r} \\
& P_{B L}=\rho_{B L}+E_{p}+b+\sigma_{p}
\end{aligned}
$$

$\rho_{B L}$ denotes the true range, $b$ denotes the phase ambiguity, and $E$ denotes the total of other errors or biases such as antenna offsets, ionospheric errors and light time delays. As in the case of GPS measurements, the RF measurements are the lower accuracy but unbiased range measurements, and the more accurate but biased carrier phase measurements. If the bias is removed and other errors are corrected, both observations will have a design matrix similar to GPS measurements:

$$
\begin{aligned}
C & =\frac{\partial c}{\partial x}=\left(\frac{\rho_{B L}}{B_{x}} \frac{\rho_{B L}}{B_{y}} \frac{\rho_{B L}}{B_{z}} 0_{1 \times 4} 0_{1 \times 2 n}\right) \\
R & =\sigma_{B L}^{2}
\end{aligned}
$$

where $n$ is the number of common-in-view satellites. The inter-satellite range measurements are combined with GPS measurements in the augmented 
design matrix:

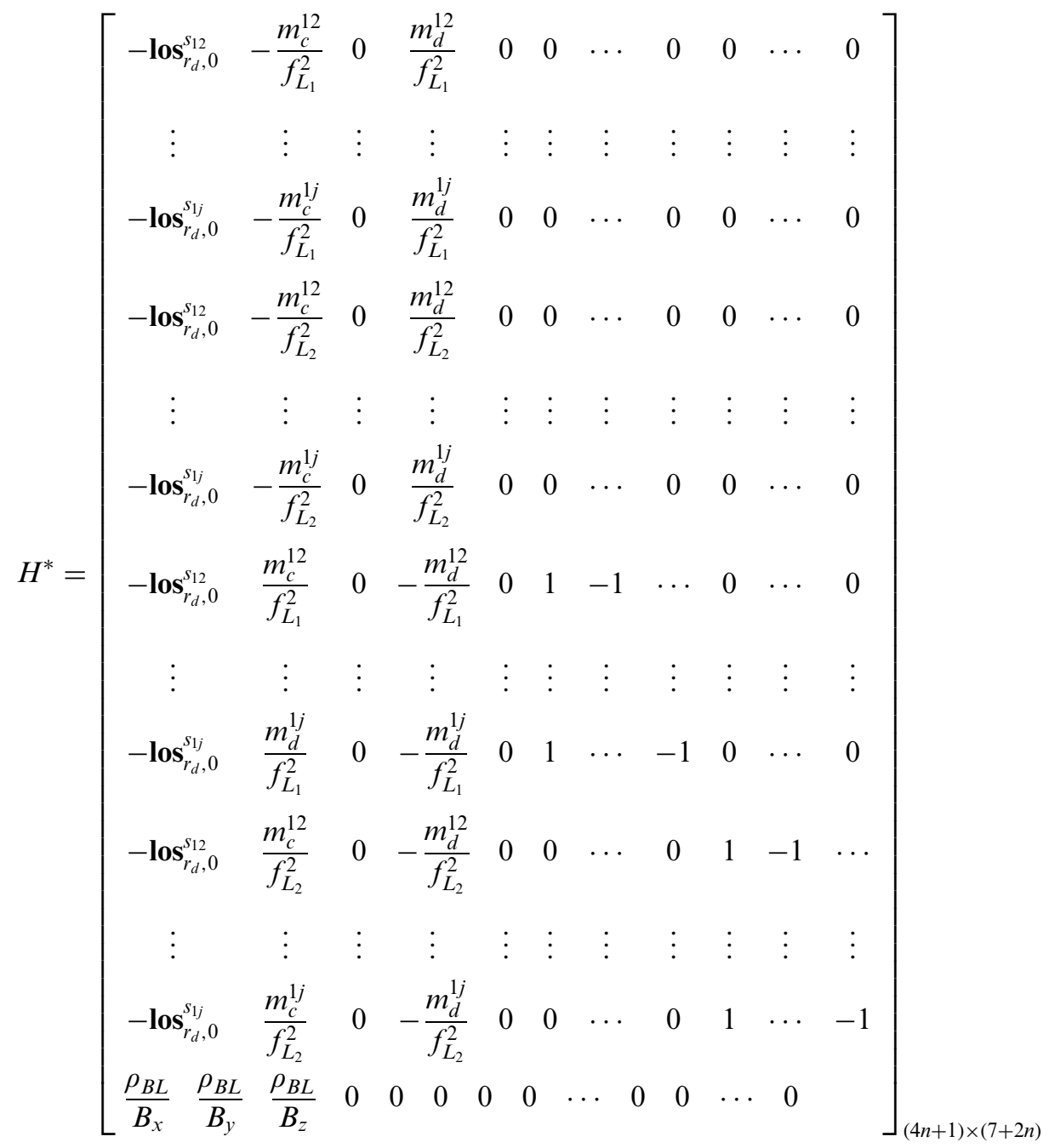

An iterative EKF strategy can be used to reduce the errors resulting from the nonlinearity of the RF measurements.

3.2. Smoothly-constrained Kalman filter. Alternatively, a SCKF can be used to process the inter-satellite measurements. In the derivation of the design matrix, the linearization is applied by truncating the Taylor series to the first order, which will result in two types of error: truncating error and base point error (De Geeter et al., 1997). In terms of the nonlinearity of the inter-satellite range measurements, a SCKF scheme is also developed in which the ARNS makes the assumption that all the intersatellite measurements are treated as equality constraints. In addition, the SCKF operates separately from the GPS EKF, as an independent module, which will contribute to a modular design of the relative navigation system. The algorithm is illustrated in Figure 1 (Simon, 2010), where $\alpha$ is a tuning parameter and $S_{t h}$ is a threshold value, both of which are empirical parameters to control the iterations of the 


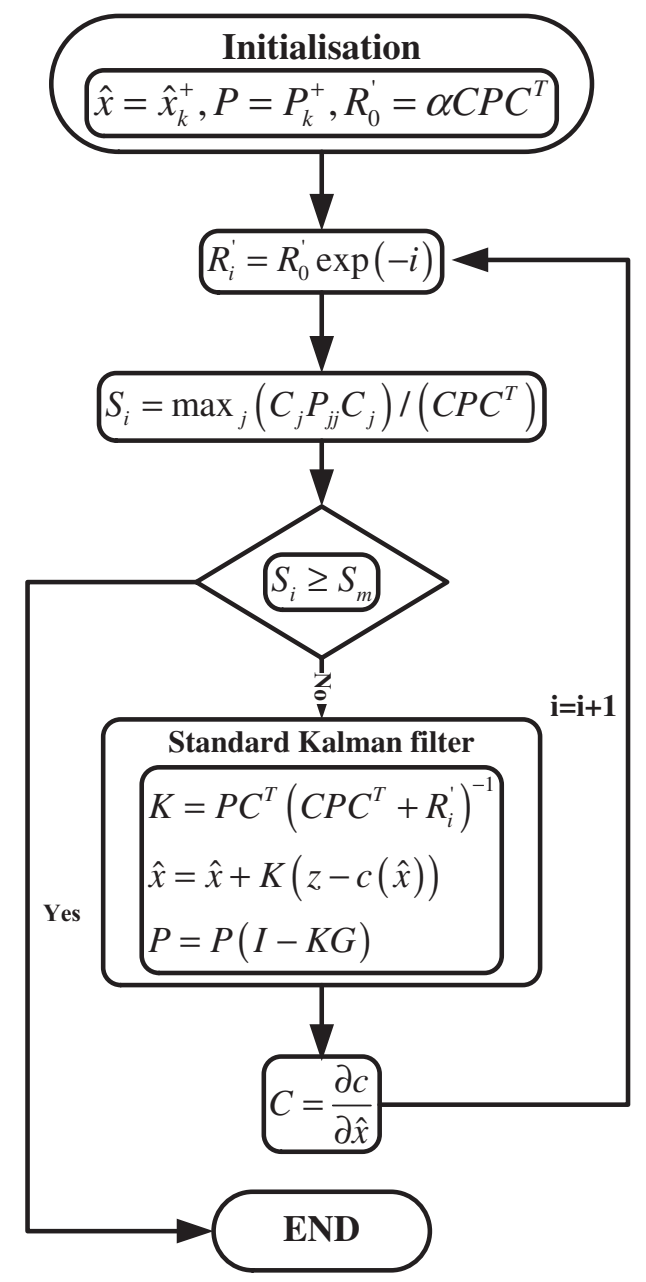

Figure 1. SCKF algorithm.

SCKF. $c$ is the constraint equation and $C$ is its Jacobian matrix as expressed in Equation (9).

\section{CARRIER PHASE INTEGER AMBIGUITY FIXING AND} FEEDBACK SCHEME. The EKF scheme mentioned in the last section gives the ambiguity float solutions of the relative states. For short baseline scenarios $(<10 \mathrm{~km})$, the float solutions have been shown to be of centimetre-level accuracy because the double-differencing operation largely removes the measurements errors (Busse, 2003). However, the integer property of the phase ambiguity should be exploited, especially when the residual ionospheric error still contaminates the doubledifferenced observables in the case of a large separation of the two spacecraft. For applications of satellite navigation/positioning, a more efficient scheme for fixing integer ambiguity is required (Kroes et al., 2005; Tancredi et al., 2013). The ambiguity float 


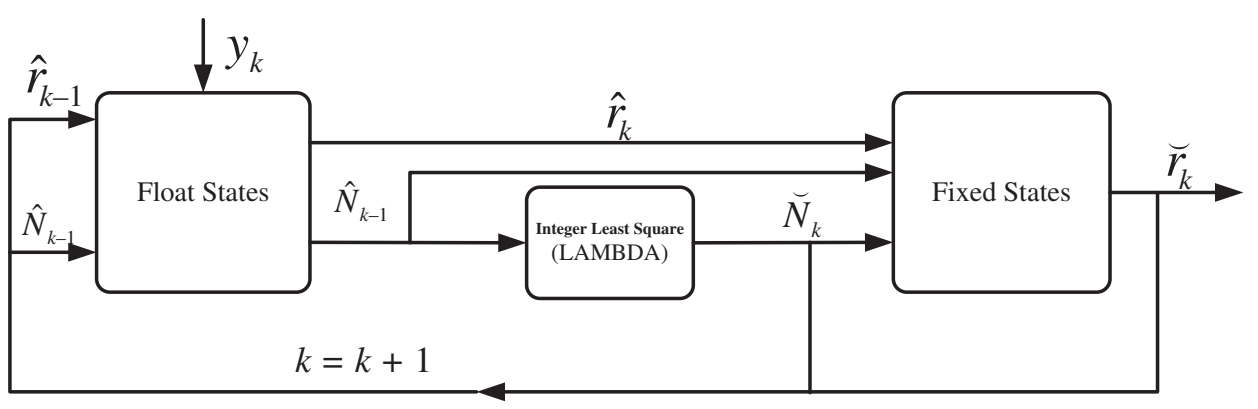

Figure 2. Fixed ambiguity feedback scheme for the GPS stand-alone relative navigation system.

solutions obtained from the EKF are used in an integer least squares (ILS) procedure, as implemented by the LAMBDA method. Once the potential integer solutions are verified in the validation step, they are adopted as pseudo-perfect measurements to be introduced into the update of the EKF for the next epoch (shown in Figure 2). A fixed ambiguity is held to an integer value until a cycle slip occurs or the filter diverges with large residuals. The pseudo-perfect measurements are formulated as:

$$
\begin{aligned}
& z=\breve{\mathbf{N}} \\
& H=\frac{\partial z}{\partial x}=\left[\begin{array}{llllllll}
0 & 0 & 0 & 0 & 0 & 0 & 0 & T
\end{array}\right] \\
& R=0
\end{aligned}
$$

where $T$ is the transformation matrix between the single-differenced ambiguities and the double-differenced ambiguities, and $R$ is the observation noise matrix which equals zero for a perfect measurement.

In the ARNS, the procedure for fixing the integer ambiguities is almost the same as in Figure 2. More specifically, when the SCKF is used to deal with the additional range measurements in the ARNS, the float relative states from the EKF are imported into the SCKF first before they are generated as fixed states, and then fed back for the next update of the EKF (Figure 3).

\section{ARNS PERFORMANCE TESTS USING GRACE FLIGHT} DATA. Real data from the GRACE mission was used to test the ARNS performance, as the two identical satellites are equipped with GPS receivers and K/KaBand Ranging (KBR) instruments. A post-processed version of the GRACE data, known as Level 1B data, was archived by JPL's Physical Oceanography Distributed Active Archive Centre (PODAAC) and is available to the science community (Case et al., 2002). PODAAC provides GPS flight data and K Band Ranging data products in the format of GPS1B and KBR1B, respectively. The Level 1B data also includes a GPS Navigation (GNV) product that contains precise orbit determination solutions of each spacecraft.

The 24 hour dataset with data interval of ten seconds, from 11 September 2005 has been selected to test the proposed ARNS. There is a gap of 30 minutes for GRACE A observation data due to receiver malfunction from epoch 3509 . Hence for relative navigation only 8460 epochs of data are valid. The separation between two satellites ranged from $20 \mathrm{~km}$ to $55 \mathrm{~km}$ during the day (Figure 4). 


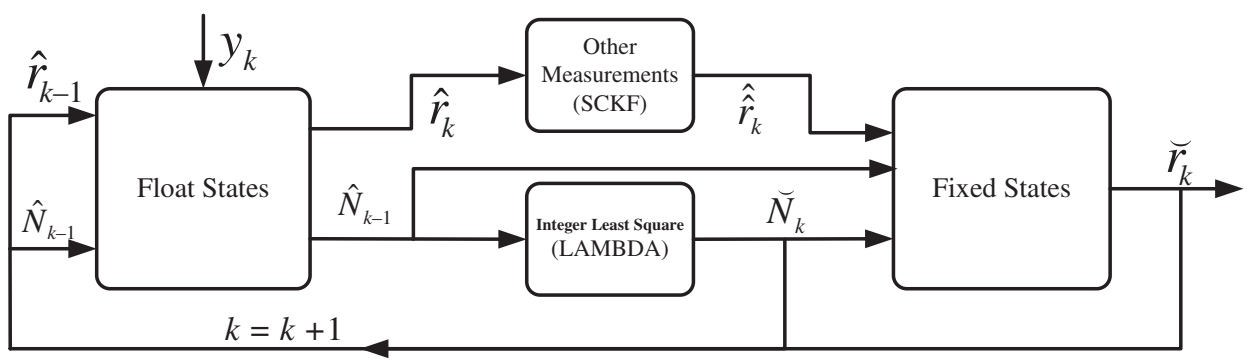

Figure 3. Fixed ambiguity feedback scheme for the SCKF-based ARNS.

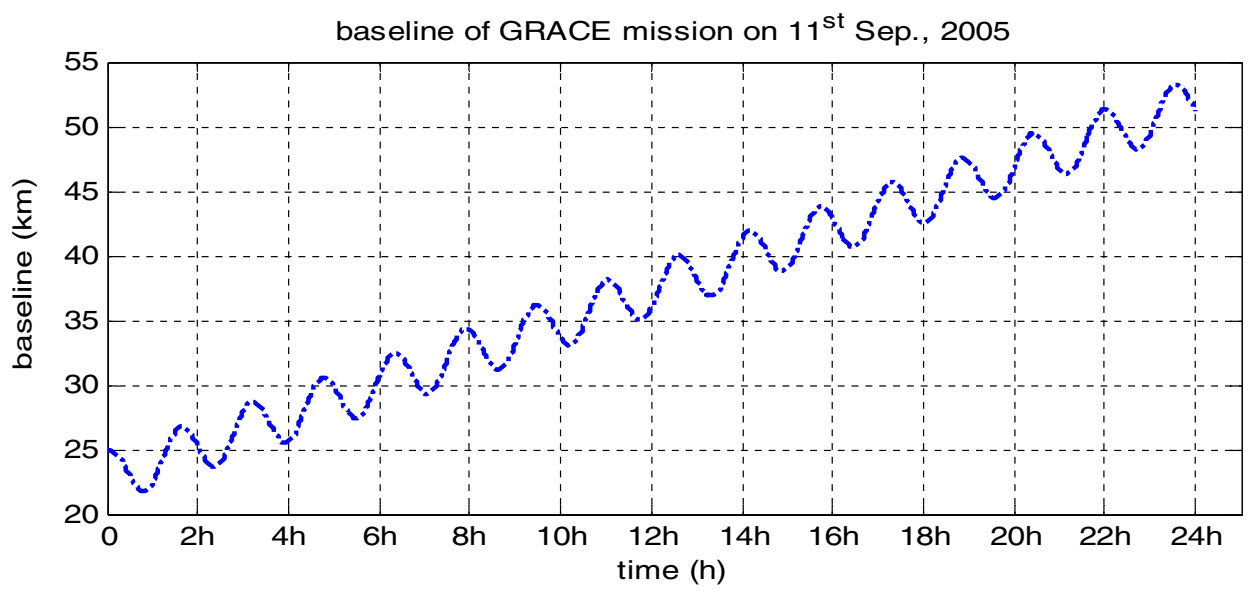

Figure 4. Inter-satellite separation for the GRACE mission.

5.1. K/Ka band range measurements. The KBR1B data are the ionosphere-free biased ranges between the GRACE A and B spacecraft. The biased range can be treated as the true range adding an unknown bias that varies at every epoch. A procedure proposed by Kroes (2006) can be used to extract the true ranges from the biased measurements. After the biases are removed, the inter-satellite range measurements can be taken as additional measurements or treated as tight constraints in the ARNS. As shown in Figure 5, the biased KBR range data is not available for all epochs, and the bias changes with time. In total there are only 6185 epochs of valid unbiased KBR data that can be processed together with double-differenced GPS observables in the ARNS.

5.2. Test results. Several tests have been conducted using the GRACE dataset. The relative positioning results are compared with the GNV products/solutions. The stand-alone GPS measurements are processed to determine the reference trajectory. The baseline estimation errors (expressed in the ECEF frame) are plotted in Figure 6. The 3D RMS error is $0.53 \mathrm{~m}$. When integrating the additional range measurements from the inter-satellite ranging system, the baseline determination accuracy improves significantly, with the 3D RMS error now being at the centimetre-level. In addition, the baseline component errors decrease to the sub-decimetre-level. Figure 7 shows the baseline error components determined using the ARNS with the SCKF algorithm.

However, as the separation distance increases, more peaks in the error occur in all three components. This confirms that long inter-satellite distances will lead to a 


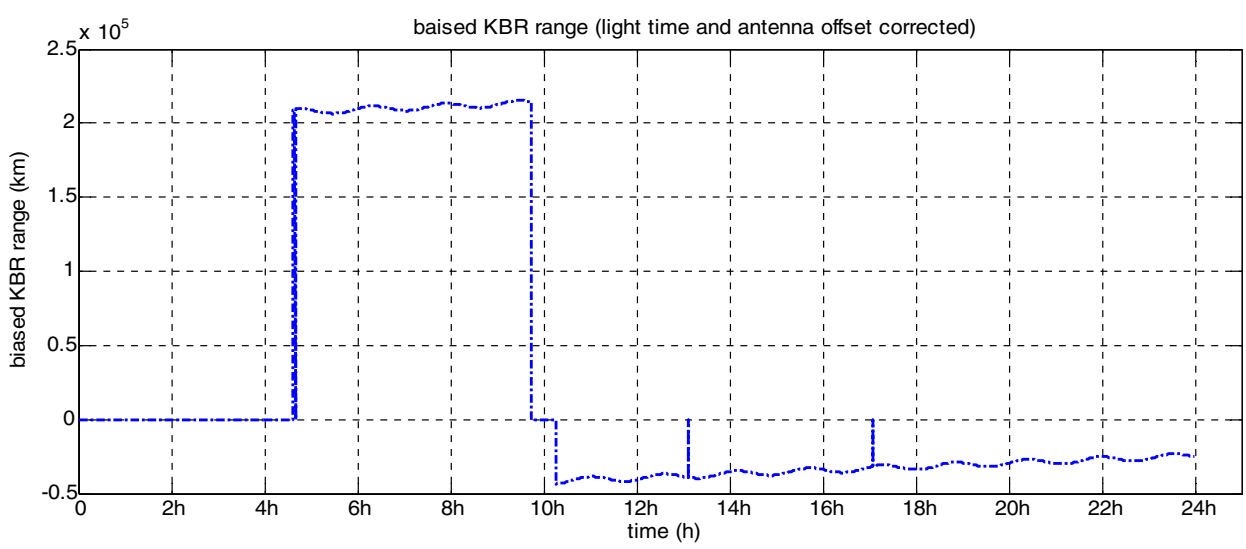

Figure 5. Biased KBR data.
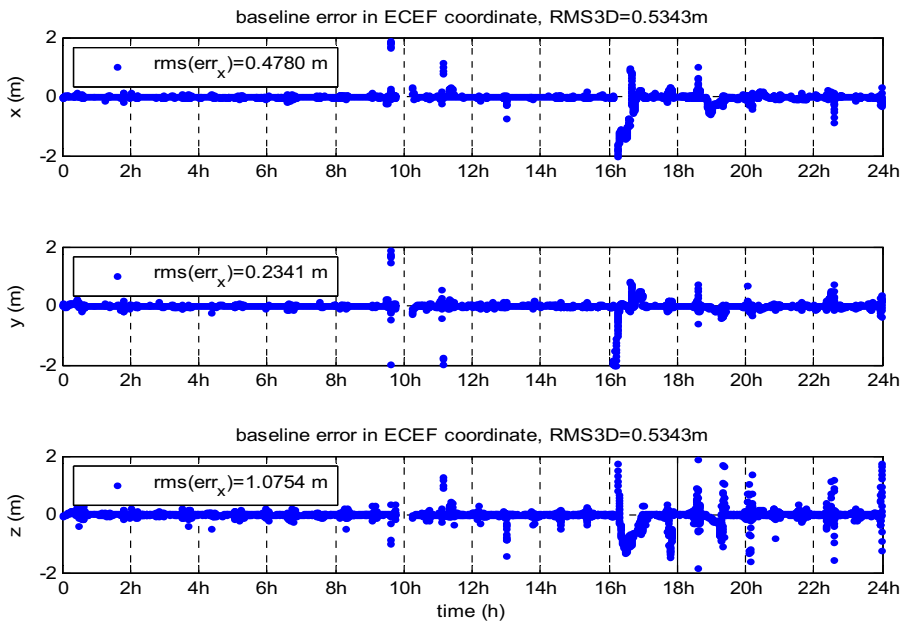

Figure 6. Baseline estimation errors for GPS stand-alone solutions.

decrease in the relative navigation performance due to de-correlated ionospheric delays, which will also worsen the phase ambiguity-fixing ratio. Figure 8 shows the vTEC values (in TEC units) for the two GPS receivers, and it can be seen clearly that the vTEC profiles of the two receivers are consistent. However, this consistency reduces as the two spacecrafts' separation increases. In Figure 9 the relative navigation solution modes of all processed epochs are plotted. The carrier phase ambiguity fixed ratio reduces with increasing inter-satellite distance.

The tests results are summarised in Table 3. The fixed phase integer ambiguity ratio benefits from the feedback strategy, which improves by about $10 \%$, from $71.0 \%$ to $81.0 \%$. This strategy also results in an improvement in the relative states estimation. The additional inter-satellite measurements in the ARNS only affect the ambiguity fixing procedure slightly, with less than $1.0 \%$ change. However, the ARNS performance has been improved significantly, by one order of baseline magnitude, in comparison with the GPS stand-alone solutions. Both methods - system 

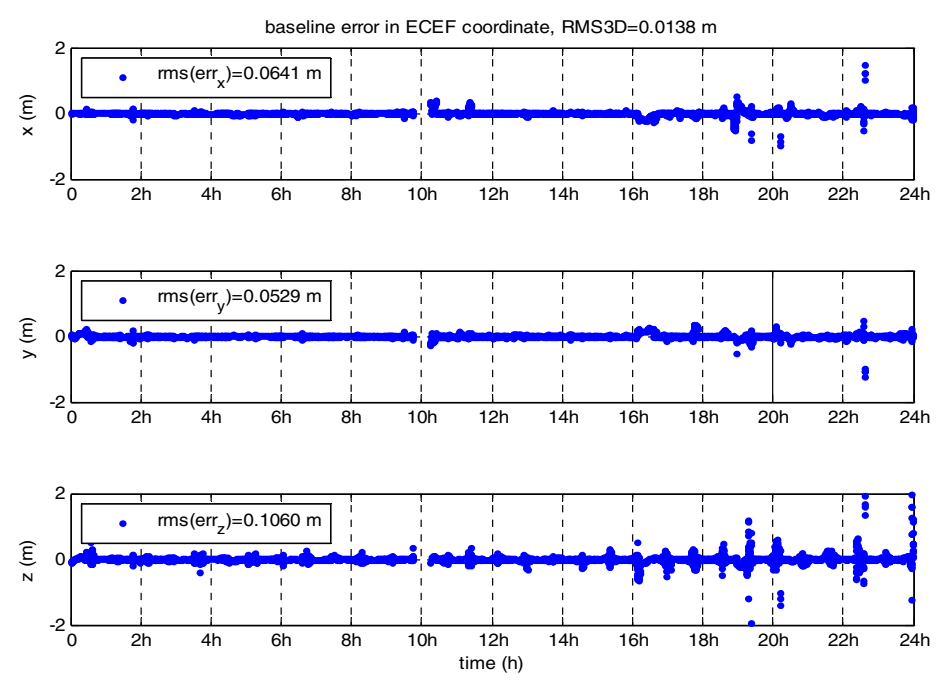

Figure 7. Baseline estimation errors for SCKF-based ARNS.

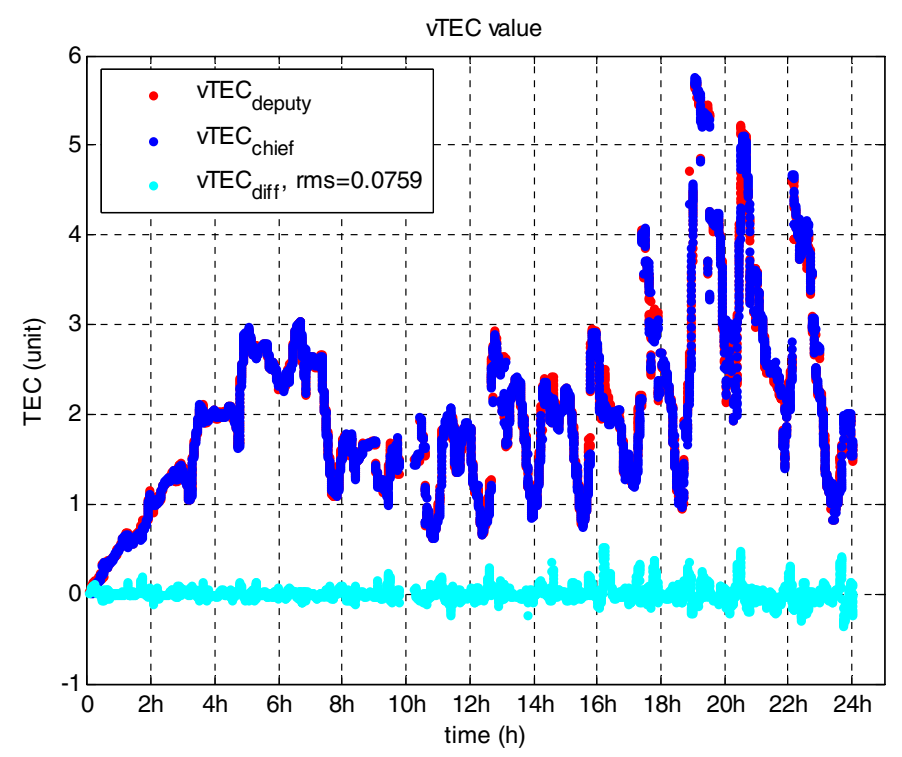

Figure 8. VTEC profiles of two spacecraft receivers.

re-parameterisation and SCKF - can be used to achieve sub-decimetre accuracy of relative state estimation in terms of the distance RMS, and centimetre accuracy for the 3D baseline estimation.

6. CONCLUDING REMARKS. An inter-satellite ranging augmented GPS relative navigation system has been presented in this paper. To combine the intersatellite measurements with GPS, two approaches were investigated. In the first method, system re-parameterisation, the original GPS stand-alone filter is augmented 
Table 3. Test results in terms of RMS error and fixed ratio value.

\begin{tabular}{|c|c|c|c|c|c|c|c|c|c|c|}
\hline & \multicolumn{5}{|c|}{ No ambiguity feedback } & \multicolumn{5}{|c|}{ Fixed ambiguity feedback } \\
\hline & \multicolumn{4}{|c|}{ RMS Error (m) } & \multirow{2}{*}{$\begin{array}{c}\text { Fixed } \\
\text { ratio (\%) }\end{array}$} & \multicolumn{4}{|c|}{ RMS Error (m) } & \multirow{2}{*}{$\begin{array}{l}\text { Fixed } \\
\text { ratio (\%) }\end{array}$} \\
\hline & $\mathrm{x}$ & $\mathrm{y}$ & $\mathrm{z}$ & $3 \mathrm{D}$ & & $\mathrm{x}$ & $\mathrm{y}$ & $\mathrm{z}$ & $3 \mathrm{D}$ & \\
\hline GPSSA & $0 \cdot 471$ & $0 \cdot 246$ & 1.075 & 0.534 & $70 \cdot 78$ & $0 \cdot 466$ & $0 \cdot 220$ & $1 \cdot 071$ & $0 \cdot 528$ & $81 \cdot 88$ \\
\hline ARNS1 & 0.065 & $0 \cdot 071$ & $0 \cdot 109$ & $0 \cdot 031$ & $70 \cdot 85$ & $0 \cdot 060$ & $0 \cdot 048$ & $0 \cdot 106$ & $0 \cdot 017$ & $81 \cdot 03$ \\
\hline ARNS2 & $0 \cdot 067$ & $0 \cdot 076$ & $0 \cdot 109$ & $0 \cdot 031$ & $70 \cdot 65$ & $0 \cdot 064$ & $0 \cdot 053$ & $0 \cdot 106$ & $0 \cdot 014$ & $81 \cdot 17$ \\
\hline
\end{tabular}

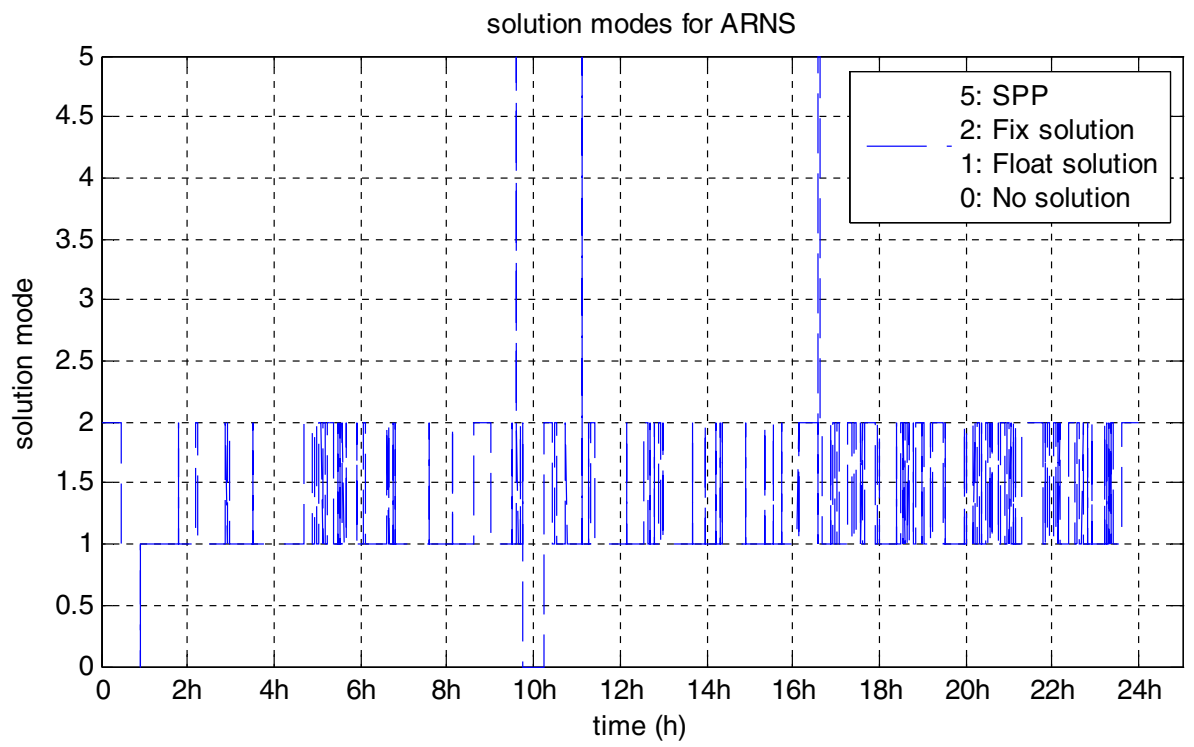

Figure 9. Relative navigation solution modes for the ARNS with fixed ambiguity feedback.

by one dimension in the observation equations. In contrast, in the second method the SCKF works in parallel with the original EKF as an independent module. Both methods can achieve significant improvement of the relative navigation performance in comparison with the GPS stand-alone solution. A phase ambiguity fixing and feedback strategy was investigated, which has been proven to increase the fix rate as well as improve the relative positioning accuracy. Real flight data from the GRACE mission was used to test the ARNS performance. The carrier-based KBR measurements were post-processed to remove the biases before they were used for relative navigation. The proposed ARNS has potential application for real-time autonomous LEO satellite formation flying missions.

\section{ACKNOWLEDGEMENT}

China Scholarship Council (CSC) is gratefully acknowledged for supporting the first author's visiting periods at University of New South Wales. 


\section{REFERENCES}

Busse, F.D. (2003). Precise Formation-state Estimation in Low Earth Orbit Using Carrier Differential GPS. Ann Arbor, Stanford University. 3085166, 192.

Case, K., Kruizinga, G. and Wu, S. (2002). "GRACE Level 1B Data Product User Handbook." JPL Publication D-22027.

D'Amico, S., Ardaens, J.S. and De Florio, S. (2013). "Autonomous formation flying based on GPS PRISMA flight results." Acta Astronautica. 82(1), 69-79.

De Geeter, J., Van Brussel, H., De Schutter, J. and Decreton, M. (1997). “A Smoothly Constrained Kalman Filter.” IEEE Transactions on Pattern Analysis and Machine Intelligence, 19(10), 1171-1177.

Jäggi, A., Montenbruck, O., Moon, Y., Wermuth, M., König, R., Michalak, G., Bock, H. and Bodenmann, D. (2012). "Inter-agency Comparison of TanDEM-X Baseline Solutions." Advances in Space Research. 50(2), 260-271.

Kroes, R. (2006). Precise Relative Positioning of Formation Flying Spacecraft Using GPS. Ann Arbor, Technische Universiteit Delft (The Netherlands). C824361, 184.

Kroes, R., Montenbruck, O., Bertiger, W. and Visser, P. (2005). "Precise GRACE Baseline Determination Using GPS.” GPS Solutions 9(1): 21-31.

Leung, S. and Montenbruck, O. (2005). "Real-Time Navigation of Formation-flying Spacecraft Using Global Positioning System Measurements." Journal of Guidance, Control, and Dynamics. 28(2), 226-235.

Mohiuddin, S. and Psiaki, M.L. (2005). Satellite Relative Navigation Using Carrier-phase Differential GPS with Integer Ambiguities. AIAA guidance, navigation, and control conference, San Francisco.

Montenbruck, O. and D'Amico, S. (2013). GPS Based Relative Navigation. Distributed Space Missions for Earth System Monitoring. M. D’Errico, Springer New York. 31, 185-223.

Montenbruck, O., Delpech, M., Ardaens, J.S., Delong, N., D'Amico, S. and Harr, J. (2008). Crossvalidation of GPS and FFRF-based Relative Navigation for the PRISMA Mission. 4th ESA workshop on satellite navigation user equipment technologies. NAVITEC.

Renga, A., Grassi, M. and Tancredi, U. (2013). "Relative Navigation in LEO by Carrier-Phase Differential GPS with Intersatellite Ranging Augmentation." International Journal of Aerospace Engineering. 2013, 11.

Sheard, B.S., Heinzel, G., Danzmann, K., Shaddock, D.A., Klipstein, W.M. and Folkner, W.M. (2012). "Intersatellite Laser Ranging Instrument for the GRACE Follow-on Mission." Journal of Geodesy. 86(12), 1083-1095.

Simon, D. (2010). "Kalman Filtering with State Constraints: a Survey of Linear and Nonlinear Algorithms." Control Theory \& Applications, IET. 4(8), 1303-1318.

Tancredi, U., Renga, A. and Grassi, M. (2010). GPS-based Relative Navigation of LEO Formations with Varying Baselines. AIAA Guidance, Navigation, and Control Conference, American Institute of Aeronautics and Astronautics.

Tancredi, U., Renga, A. and Grassi, M. (2013). "Validation on Flight Data of a Closed-loop Approach for GPS-based Relative Navigation of LEO Satellites.” Acta Astronautica. 86, 126-135.

Tapley, B.D., Bettadpur, S., Ries, J.C., Thompson, P.F. and Watkins, M.M. (2004). GRACE Measurements of Mass Variability in the Earth System. Science. 305(5683), 503-505.

van Barneveld, P., Montenbruck, O. and Visser, P. (2008). Differential Ionospheric Effects in GPS Based Navigation of Formation Flying Spacecraft. Proceedings of 3rd international symposium on formation flying, missions and technology, ESAIESTEC, Noordwijk. 